\title{
A Characterization of Cylinders and an Estimate for Mean Curvature of Convex Euclidean Hypersurfaces Satisfying a Cylindrical Condition
}

\author{
Leonard M. Giugiuc and Bogdan D. Suceavă* \\ (Communicated by Bang-Yen Chen)
}

\begin{abstract}
We show that a curvature condition on the Gauss-Kronecker curvature and scalar curvature of a convex smooth hypersurface lying in the four dimensional Euclidean space yields a lower bound for the mean curvature. The curvature condition we investigate is suggested by the local geometry of cylinders in the four dimensional Euclidean space.
\end{abstract}

\section{Introduction}

Myers proved in 1941 [17] an important result providing sufficient conditions in terms of Ricci curvature to determine the compactness of a Riemannian manifold. His work was inspired by Ossian Bonnet's original inquiry [1]. This theorem inspired many authors e.g. [5, 13], more recently [2], and attracted the interest on prescribing meaningful curvature conditions.

The classical curvature invariants are the Gaussian curvature [14] and Sophie Germain's mean curvature [15], which are studied in literature, see e.g. [12]. By the other hand, B.-Y. Chen introduced in the early 1990s a class of fundamental curvature invariants $[6,7,9]$, which raise many other interesting questions in the general area of geometric inequalities. These fundamental works motivated and inspired several other constructions e.g., among many other papers, $[4,10,11,18,19,20,21]$, etc. For a complete overview of the developments, the best reference is [9]. The inspiration in our study is the context created by these recent developments.

The inequality discussed in this paper pursues a recent result published by the first author in [16]. We can view the present discussion as a quest for the geometric interpretation of a natural algebraic inequality, in the spirit of $[3,20]$.

\subsection{Notations in the Geometry of Smooth Hypersurfaces}

To recall a few concepts in the differential geometry of smooth hypersurfaces, we start by considering $\sigma: U \subset$ $\mathbb{R}^{n} \rightarrow \mathbb{R}^{n+1}$, a smooth hypersurface given by the smooth map $\sigma$. Let $p$ be a point on the hypersurface. Denote $\sigma_{k}(p)=\frac{\partial \sigma}{\partial x_{k}}$, for all $k$ from 1 to $n$. Consider $\left\{\sigma_{1}(p), \sigma_{2}(p), \ldots, \sigma_{n}(p), N(p)\right\}$, the Gauss frame of the hypersurface, where $N$ denotes the normal vector field. We denote by $g_{i j}(p)$ the coefficients of the first fundamental form and by $h_{i j}(p)$ the coefficients of the second fundamental form. Then

$$
g_{i j}(p)=\left\langle\sigma_{i}(p), \sigma_{j}(p)\right\rangle, \quad h_{i j}(p)=\left\langle N(p), \sigma_{i j}(p)\right\rangle .
$$


The Weingarten map $L_{p}=-d N_{p} \circ d \sigma_{p}^{-1}: T_{\sigma(p)} \sigma \rightarrow T_{\sigma(p)} \sigma$ is linear. Denote by $\left(h_{j}^{i}(p)\right)_{1 \leq i, j \leq n}$ the matrix associated to Weingarten's map, that is:

$$
L_{p}\left(\sigma_{i}(p)\right)=h_{i}^{k}(p) \sigma_{k}(p),
$$

where the repeated index and upper script above indicates Einstein's summation convention. Weingerten's operator is self-adjoint, which implies that the roots of the algebraic equation

$$
\operatorname{det}\left(h_{j}^{i}(p)-\lambda(p) \delta_{j}^{i}\right)=0
$$

are real. The eigenvalues of Weingarten's linear map are called principal curvatures of the hypersurface. They are the roots $k_{1}(p), k_{2}(p), \ldots, k_{n}(p)$ of this algebraic equation. The mean curvature at the point $p$ is

$$
H(p)=\frac{1}{n}\left[k_{1}(p)+\ldots+k_{n}(p)\right]
$$

and the Gauss-Kronecker curvature is

$$
K(p)=k_{1}(p) k_{2}(p) \ldots k_{n}(p) .
$$

If all the principal curvature of a smooth regular hypersurface are $\geq 0$, then the hypersurface is convex.

\section{The Main Result}

We start our discussion by investigating an example. Consider the cylinder $S^{1} \times \mathbb{R} \times \mathbb{R} \subset \mathbb{R}^{4}$, given by the parametrization

$$
\sigma(t, y, z)=<\cos t, \sin t, y, z>
$$

Then its principal curvatures are $a=1$, and $b=c=0$ at every point. It is natural to think of extending this description of cylinders by the local behavior of the hypersurface's principal curvatures. The idea we pursue is the following.

Let $\sigma: U \subset \mathbb{R}^{3} \rightarrow \mathbb{R}^{4}$ be a smooth hypersurface and let $p$ be a point on the hypersurface. We denote by $a, b, c$ the principal curvatures at $p$. By using Gauss formula, we have $\operatorname{scal}(p)=a b+b c+c a$. The mean curvature is $H(p)=\frac{1}{3}(a+b+c)$, and the Gauss-Kronecker curvature is $K(p)=a b c$.

The curvature condition we investigate is

$$
(1-a)(1-b)(1-c)=a b c .
$$

Remark first that the condition (2.1) is equivalent to

$$
1+a b+b c+c a=a+b+c+2 a b c
$$

which translates in terms of curvature invariants into

$$
1+s c a l=3 H+2 K
$$

We prove the following result, whose geometric interpretation will be discussed right after the proof.

Theorem 2.1. Let $\sigma: U \subset \mathbb{R}^{3} \rightarrow \mathbb{R}^{4}$ be a smooth convex hypersurface and let $p$ be a point on the hypersurface. Let $a, b, c$ be the principal curvatures at $p$. Suppose $\operatorname{scal}(p)=a b+b c+c a$, the mean curvature $H(p)=\frac{1}{3}(a+b+c)$, and the Gauss-Kronecker curvature $K(p)=$ abc satisfy the condition

$$
1+s c a l=3 H+2 K
$$

Then

$$
3 H(p) \geq 1 .
$$

Equality holds at parabolic points where the principal curvatures are two of them zeroes and one of them equal to 1. 
Proof: Should either of the principal curvatures $a, b$, or $c$ be zero, then at least one of the other principal curvatures must be 1 , and this proves the assertion. If e.g. $a=0$ and $b=1$, then the equality case in

$$
3 H(p)=1
$$

yields immediately $c=0$, so the parabolic points with principal curvature $(1,0,0),(0,1,0)$, and $(0,0,1)$ satisfy the equality case. It is actually the only case in which the equality holds, as we shall soon see.

If one of the principal curvatures $a, b$, or $c$ is greater than 1 , then the conclusion follows immediately.

We need to prove the statement for $0<a, b, c<1$. We will prove the inequality holds as claimed, it will be strict, and the equality can't occur.

We prove first the following assertion. If $0<a, b, c<1$ satisfy $(1-a)(1-b)(1-c)=a b c$, then there exist the real positive numbers $x, y, z$ such that

$$
a=\frac{x}{x+y}, \quad b=\frac{y}{y+z}, \quad c=\frac{z}{z+x} .
$$

To prove this assertion, consider $z>0$ a fixed arbitrary real number. From $b=\frac{y}{y+z}$, it follows immediately that $y=\frac{b z}{1-b}>0$, and from $a=\frac{x}{x+y}$ it follows that

$$
x=\frac{a b z}{(1-a)(1-b)}>0 .
$$

Since $(1-a)(1-b)(1-c)=a b c$, then $\frac{z}{z+x}=c$. This proves the assertion.

For our second step, we claim that for $x, y, z>0$, the following inequality always holds

$$
\frac{x}{x+y}+\frac{y}{y+z}+\frac{z}{z+x}>1 .
$$

To prove this inequality, we use the substitutions $u=\frac{y}{x}, v=\frac{z}{y}$, and $w=\frac{x}{z}$. We have $u, v, w>0$, and $u v w=1$. Furthermore,

$$
\frac{x}{x+y}+\frac{y}{y+z}+\frac{z}{z+x}=\frac{1}{1+u}+\frac{1}{1+v}+\frac{1}{1+w} .
$$

Then the inequality (2.2) is equivalent to

$$
\frac{1}{1+u}+\frac{1}{1+v}+\frac{1}{1+w}>1
$$

which can be expanded directly to

$$
3+2(u+v+w)+u v+v w+w u>1+u+v+w+u v+v w+w u+1,
$$

and this last inequality reduces to $1+u+v+w>0$, which holds true. Note that in this case we can not have equality.

Remark that cylinders $S^{1} \times \mathbb{R} \times \mathbb{R} \subset \mathbb{R}^{4}$ satisfy the equality case at every point on the surface.

\subsection{Conclusion}

In her work published in 1831, Sophie Germain [15] investigated the properties of the mean curvature and presented a solid argument on the importance of the study of mean curvature. Her motivation was triggered by "la nécessité de savoir exprimer d'une manière générale les résistences nées de la courbure des surfaces." Sophie Germain's original intuition was to compare the curvature of spherical surfaces with that of cylindrical surfaces. With this thought in mind, the Theorem we stated and proved above can be formulated in the following way: if the hypersurface satisfies a cylinder-like curvature hypothesis, then the tension expressed by the mean curvature can not be small. This is an illustration of Sophie Germain's intuition for three-dimensional smooth hypersurfaces in $\mathbb{R}^{4}$. Sophie Germain's original example was the cylinder, for which she pointed out that the Gaussian curvature vanishes at every point, but to our intuition it looks curved [15]. 


\section{References}

[1] Bonnet, O., Sur quelque propriétés des lignes géodésiques. Comptes rendus de l'Academie des Sciences 11 (1855), 1311-1313.

[2] Brubaker, N.D. and Suceavă, B.D., A Geometric Interpretation of Cauchy-Schwarz Inequality in Terms of Casorati Curvature. Intern. Elec. J. Geom. 11 (2018), 48-51.

[3] Brubaker, N.D., Camero, J., Rocha Rocha O., Soto, R. and Suceavă, B.D., A Curvature Invariant Inspired by Leonhard Euler's Inequality $R \geq 2 r$. Forum Geometricorum 18 (2018) 119-127.

[4] Brzycki, B., Giesler, M.D., Gomez, K., Odom L.H. and Suceavă, B.D., A ladder of curvatures for hypersurfaces in the Euclidean ambient space. Houston J. Math. 40 (2014) 1347-1356.

[5] Calabi, E., On Ricci curvatures and geodesics. Duke Math. J. 34 (1967), 667-676.

[6] Chen, B.-Y., Some pinching and classification theorems for minimal submanifolds. Arch. Math. 60 (1993), 568-578.

[7] Chen, B.-Y., A Riemannian invariant and its applications to submanifold theory. Results Math. 27 (1995), 17-26.

[8] Chen, B.-Y., Some new obstructions to minimal and Lagrangian isometric immersions. Japanese J. Math. 26 (2000), 105-127.

[9] Chen, B.-Y., Pseudo-Riemannian submanifolds, $\delta$-invariants and Applications. World Scientific, 2011.

[10] Conley, C. T. R., Etnyre, R., Gardener, B., Odom L.H. and Suceavă, B.D., New Curvature Inequalities for Hypersurfaces in the Euclidean Ambient Space. Taiwanese J. Math. 17 (2013), 885-895.

[11] Dillen, F., Fastenakels, J. and Van der Veken, J., A pinching theorem for the normal scalar curvature of invariant submanifolds. J. Geom. Phys. 57 (2007), no. 3, 833-840.

[12] doCarmo, Manfredo P., Riemannian Geometry. Birkhäuser, 1992.

[13] Galloway, G.J., A Generalization of Myers Theorem and an application to relativistic cosmology. J. Diff. Geom. 14 (1979), $105-116$.

[14] Gauss, C.F. , Disquisitiones circa superficies curvas. Typis Dieterichianis, Goettingen, 1828.

[15] Germain, S., Mémoire sur la courbure des surfaces. J. Reine Angew. Math. 8 (1832), 280-297.

[16] Giugiuc, L.M., Problem 11911. American Mathematical Monthly 123 (2016), p. 504.

[17] Myers, S.B., Riemmannian manifolds with positive curvature. Duke Math. J. 8 (1941), 401-404.

[18] Suceavă, B. D., The spread of the shape operator as conformal invariant. J. Inequal. Pure Appl. Math. 4 (2003), article 74.

[19] Suceavă, B. D., The amalgamatic curvature and the orthocurvatures of three dimensional hypersurfaces in $\mathbb{E}^{4}$. Publicationes Mathematicae 87 (2015), no. 1-2, 35-46.

[20] Suceavă, B.D., A Geometric Interpretation of Curvature Inequalities on Hypersurfaces via Ravi Substitutions in the Euclidean Plane. Math. Intelligencer 40 (2018), 50-54.

[21] Suceavă, B.D. and Vajiac, M. B. Remarks on Chen's fundamental inequality with classical curvature invariants in Riemannian spaces. An. Ştiinţ. Univ. Al. I. Cuza Iaşi. Mat. (N.S.) 54 (2008), no. 1, 27-37.

\section{Affiliations}

LEONARD M. GIUGIUC

AdDreSS: Bd. Carol I No. 6, National College Trajan, Drobeta-Turnu Severin, Romania.

E-MAIL: leonardgiugiuc@yahoo.com

ORCID ID : orcid.org/0000-0003-0425-7762

BOGDAN D. SUCEAVĂ

Address: 800 N. State College Blvd., Department of Mathematics, California State University at Fullerton, Fullerton, CA 92834-6850, U.S.A.

E-MAIL: bsuceava@fullerton.edu

ORCID ID : orcid.org/0000-0003-3361-3201 